\title{
Assessment of Potential ICT-Related Collaboration and Innovation Capacity in East Africa
}

\author{
Paul Cunningham \\ IIMC / IST-Africa / Department of \\ Computer and Systems Sciences, \\ DSV, Stockholm University \\ 13 Docklands Innovation Park \\ 128 East Wall Rd, Dublin3, Ireland \\ +353-1-817 0607, paul@iimg.com
}

\author{
Miriam Cunningham \\ IIMC / IST-Africa / Department of \\ Computer and Systems Sciences, \\ DSV, Stockholm University \\ 13 Docklands Innovation Park \\ 128 East Wall Rd, Dublin3, Ireland \\ +353-1-817 0607, miriam@iimg.com
}

\author{
Love Ekenberg \\ International Institute for Applied \\ Systems Analysis (IIASA) \\ Schlossplatz 1 - A-2361 Laxenburg, \\ Austria \\ +43 (0) 2236807338 \\ ekenberg@iiasa.ac.at
}

\begin{abstract}
Due to a significant investment in digital infrastructure and a pro-innovation policy and regulatory framework, the Innovation Ecosystems in Nairobi, Dar es Salaam and Kampala have considerably expanded over the last five years, incorporating new national and international Innovation Stakeholders. This is important in the context of realising the objectives of National Development Plans, and addressing high levels of youth and graduate unemployment. This paper presents a sub-set of results from a comprehensive baseline analysis of Innovation Ecosystems in these cities with a focus on assessing the current level of ICT-related Collaboration, Innovation Absorption capacity and challenges to be addressed. In order to benefit from these developments, it is recommended that the public sector take a leadership role in establishing necessary mechanisms that will stimulate multi-stakeholder collaboration amongst existing Innovation Actors to foster a sustainable Collaborative Open Innovation and Entrepreneurial culture.
\end{abstract}

Keywords - Collaborative Open Innovation, National Innovation Ecosystem, ICT4D, Research, Entrepreneurship, SocioEconomic Impact

\section{INTRODUCTION}

As the basis of any development theory, people must have a vital role. "People are the real wealth of a nation. The basic objective of development is to create an enabling environment for people to enjoy long, healthy and creative lives. This may appear to be a simple truth. But it is often forgotten in the immediate concern with the accumulation of commodities and financial wealth. The basic purpose of development is to enlarge people's choices. In principle, these choices can be infinite and can change over time. People often value achievements that do not show up at all, or not immediately, in income or growth figures." [17]. Such ideas are to a large extent contrasting against earlier growth-focused perspectives and many have tried to define alternatives to such. One of the more well-known is Sen's Capabilities Approach [13] having a significant influence on the UN Human Development Report [14] introducing the Human Development Index as a method to measure development with literacy, life expectancy and standard of living added as indicators.

However, Sen's Capabilities Approach is general and problematic when it comes to details. [11, p35] highlighted the limited use of Sen's Approach for impact assessment in ICT4D (ICT for Development) projects since "capabilities are both inputs to and outputs from any ICT4D project". Sen's ideas must be made more precise to be meaningful, and, e.g., [12] focuses on how to operationalise Sen's Capabilities Approach in the ICT4D domain, looking at challenges associated with "granularity of the capability approach" [para. 1]. Heeks extrapolated this requires development to be broken down "not merely to the level of individuals but to the level of single capabilities or functionings" [para. 1]. While [12] acknowledged that [1] and [15] explored capabilities approach operationalization through aggregation, he proposes an alternative type of aggregation based on a user-role based approach, assigning specific tasks and behaviours to roles adopted in ICT engagement. [12] argued that "roles therefore represent something halfway between a realised functioning and a livelihood" [para. 3]. [13] proposes a Ladder of ICTrelated Roles. The further up the ladder (from Intermediated consumer, Passive Consumer, Active User, Producer, Worker, Entrepreneur to Innovator), the greater the level of ICT engagement, technological competency and realised functioning. While acknowledging this adaptation of Sen's approach, he suggests this offers a practical way of evaluating ICT4D projects leveraging the intentions of the Capabilities Approach whereby each successive role is "one more step in the climb away from poverty" (p19). [13, p22] points out that in Africa higher-level roles are associated with mobile usage: "mobile users are at least passive users; and increasing numbers will be active users and even producers". [13, p26] highlights that "ICT innovators are creating a complete new livelihood and new role for themselves that can radically alter their poverty status". This combines well with the learningcentered approach from [14] and the ladder idea is well aligned with the primary findings of this study - that active stakeholder collaboration is required to strengthen overall Innovation Ecosystems. The Ladder of ICT-related roles facilitate stakeholder and community segmentation to better understand the capacity of Innovation Ecosystem Stakeholders and providing a roadmap towards expanding peoples' choices.

The paper focuses on comparing research findings in the target cities with existing literature, and particularly the $7^{\text {th }}$ Global Innovation Index (GII 2014). Section 2 provides insight from the GII 2014. Section 3 outlines the study methodology. Sections 4, 5 and 6 present a snapshot of key Innovation Ecosystem Stakeholders in target cities, and a qualitative assessment of ICT-related collaboration. Section 7 presents a 
synthesis of the absorption capacity of innovation stakeholders and common challenges to be addressed. Section 8 presents a summary of key findings and compares them with literature. Section 9 presents conclusions and recommendations.

\section{GLOBAL INNOVATION INDEX FRAMEWORK}

GII 2014 [9] covers 143 economies, accounting for $92.9 \%$ of global population and $98.3 \%$ of GDP. [7] is based on primarily quantitative data from over 30 sources. As the underlying framework is regularly revised based on lessons learnt from previous years, annual scores and rankings are not directly comparable. GII is based on two sub-indices (Innovation Input Sub-Index, Innovation Output Sub-Index) built on Input Pillars (Institutions, Human Capital and Research, Infrastructure, Market Sophistication, Business Sophistication) and Output Pillars (Knowledge and Technology Outputs, Creative Outputs). GII is the average of Input and Output Sub-Indices. The Innovation Input Sub-Index is the average of the first five pillar scores, while the Innovation Output Sub-Index is the average of the last two pillar scores. Individual pillar scores are calculated as weighted averages of sub-pillar scores, which in turn are calculated as the weighted average of individual indicators. The Innovation Efficiency Ratio is Output divided by Input Sub-Index. While generally, the higher the Innovation Efficiency Ratio, the better, this should be considered in an overall context. A relatively high efficiency ratio can result from a very low input score. The GII 2014 theme is "Human Factor in Innovation", which "explores the role of the individuals and teams behind the innovation process." [6, Preface] Chapter 1 notes that to identify relevant strategies and polices that energise innovators "it is important to learn more about what happens at the intersection of people, technology, financing, policy and institutions". Chapter 3 notes that "Improving skills is one of the most important ways to raise innovation, productivity and economic growth."

\section{A. Relevant GII Results in Africa}

Sub-Saharan Africa is noted as achieving the most significant improvement in 2014 GII rankings, with 2 new countries and 17 of 33 countries represented improving rank. While GII results are national in focus, they still provide useful background comparative data to inform discussion and analysis of ICT-related Collaboration and Innovation Absorption Capacity in Nairobi, Dar es Salaam and Kampala, where, Uganda and Kenya have achieved comparable overall GII 2014 overall rank and scores compared to Tanzania.

Within Low Income Economies, Kenya is ranked first, Uganda second, while Tanzania lags significantly behind. There are also significant differences in relative Innovation Efficiency Rank: Kenya (rank 26); Uganda (rank 77); and Tanzania (rank 113). While Uganda achieves a slightly better Innovation Input Sub-Index Score to Kenya (ranked fourth and fifth of Low Income Economies), Kenya achieves a significantly higher Innovation Output Sub-Index Score than Uganda (ranking first and second of Low Income Economies). Tanzania's low Innovation Efficiency Rank (113) is explained by its significantly lower Innovation Input and Output SubIndex Scores, compared to Kenya (26) and Uganda (77), which are ranked first and sixth of Low Income Economies.
GII classifies Kenya and Uganda as Innovation Learners (countries where innovation is increasing due to improvements in institutional frameworks, innovation infrastructure, skilled labour force and business environment), Kenya is classified as an Efficient Innovator, while Uganda and Tanzania are Inefficient Innovators (Innovation Efficiency Ratios < 0.74).

Table 1. Comparison based on GII 2014 Analysis of Pillar 2

\begin{tabular}{|l|c|c|c|}
\hline \multicolumn{1}{|c|}{ GII 2014 (Pillar 2) } & Kenya & Tanzania & Uganda \\
\hline $\begin{array}{l}\text { Human Capital and } \\
\text { Research Rank }\end{array}$ & 117 & 132 & $\mathbf{1 1 4}$ \\
\hline $\begin{array}{l}\text { Human Capital and } \\
\text { Research Score }\end{array}$ & 15.8 & 12.7 & $\mathbf{1 7 . 3}$ \\
\hline Education Sub-Pillar Rank & $\mathbf{9 4}$ & 115 & 109 \\
\hline Education Sub-Pillar Score & $\mathbf{3 5 . 9}$ & 30.8 & 31.9 \\
\hline $\begin{array}{l}\text { Tertiary Education Sub- } \\
\text { Pillar Rank }\end{array}$ & 134 & 135 & $\mathbf{1 1 0}$ \\
\hline $\begin{array}{l}\text { Tertiary Education Sub- } \\
\text { Pillar Score }\end{array}$ & 3.3 & 3.2 & $\mathbf{1 5 . 5}$ \\
\hline R\&D Sub-Pillar Rank & $\mathbf{7 3}$ & 89 & 86 \\
\hline R\&D Sub-Pillar Score & $\mathbf{8 . 4}$ & 4.0 & 4.4 \\
\hline
\end{tabular}

In addition, Pillar 2 (Human Capital and Research) is focused on assessing the human capital of countries, based on achievements at primary/elementary, secondary/high school and higher education/university level and the quality and level of R\&D activities, Table 1 illustrates that based on GII 2014 data, Uganda and Kenya have achieved comparable overall rank and scores for Pillar 2 compared to Tanzania, which lags further behind. However, an analysis of relative strengths at a sub-pillar level highlights significant differences, with Uganda achieving a significantly stronger score for Tertiary Education while Kenya achieved a stronger score for Education (Primary and Secondary) and a significantly stronger score for R\&D.

Finally, Pillar 5 (Business Sophistication) links "productivity, competitiveness and innovation potential with the employment of highly qualified professionals and technicians." (GII 2014, p49) In this respect, Uganda ranks significantly higher than Kenya and Tanzania. While overall rank and score for Kenya and Tanzania shows a tight range, at a sub-pillar level Tanzania outperformed Kenya for Knowledge Workers and Innovation Linkages, but was significantly weaker in Knowledge Absorption.

\section{MethodOLOGY}

Face to face semi-structured interviews were selected as the most appropriate data collection method. In terms of sampling, non-probability and purposive sampling were selected to identify respondents that could provide new insights based on expertise and experience [7, p.24, p.34] including (a) accessing highly fragmented or not publicly available intelligence; (b) gaining insight into decision making processes influencing policy and implementation; and (c) informed feedback on proposed solutions. Interviews were up to two hours long, carried out by two interviewers, one asking semi-structured questions, the other making detailed notes. Interviewees also 
shared insight into other issues they felt were important. Table 2 below provides insight into the range of respondents:

Table 2

Key Innovation Stakeholders Interviewed

\begin{tabular}{|l|c|c|c|}
\hline Stakeholders & Nairobi & Kampala & Dar es Salaam \\
\hline $\begin{array}{l}\text { Independent } \\
\text { Innovation Spaces }\end{array}$ & 2 & 5 & 1 \\
\hline $\begin{array}{l}\text { University } \\
\text { Innovation Spaces }\end{array}$ & 3 & 2 & 3 \\
\hline $\begin{array}{l}\text { Government } \\
\text { Innovation Spaces }\end{array}$ & 1 & 1 & 2 \\
\hline NREN & 1 & 1 & 1 \\
\hline ICT Regulator & 3 & 2 & 1 \\
\hline Public Sector & & & 3 \\
\hline
\end{tabular}

Data was analysed following Creswell's Data Analysis Spiral [3, p.151]. Direct data collection was supplemented by desk research, annual IST-Africa surveys of Research and Innovation capacities [6] and ICT/STI related Bilateral and Multilateral Cooperation [5], insight captured during 6 one day IST-Africa Workshops (2011 - 2014), and e-mail and phone interviews to reflect developments to July 2014. This paper compares these research findings with literature findings.

\section{NAIROBI INNOVATION ECOSYSTEM}

Starting with Kenya, there are a good range of stakeholders supporting Innovation and Entrepreneurship in Nairobi (Funding, Education and Research, NREN, Innovation Spaces, Public, Private and Societal Sectors).

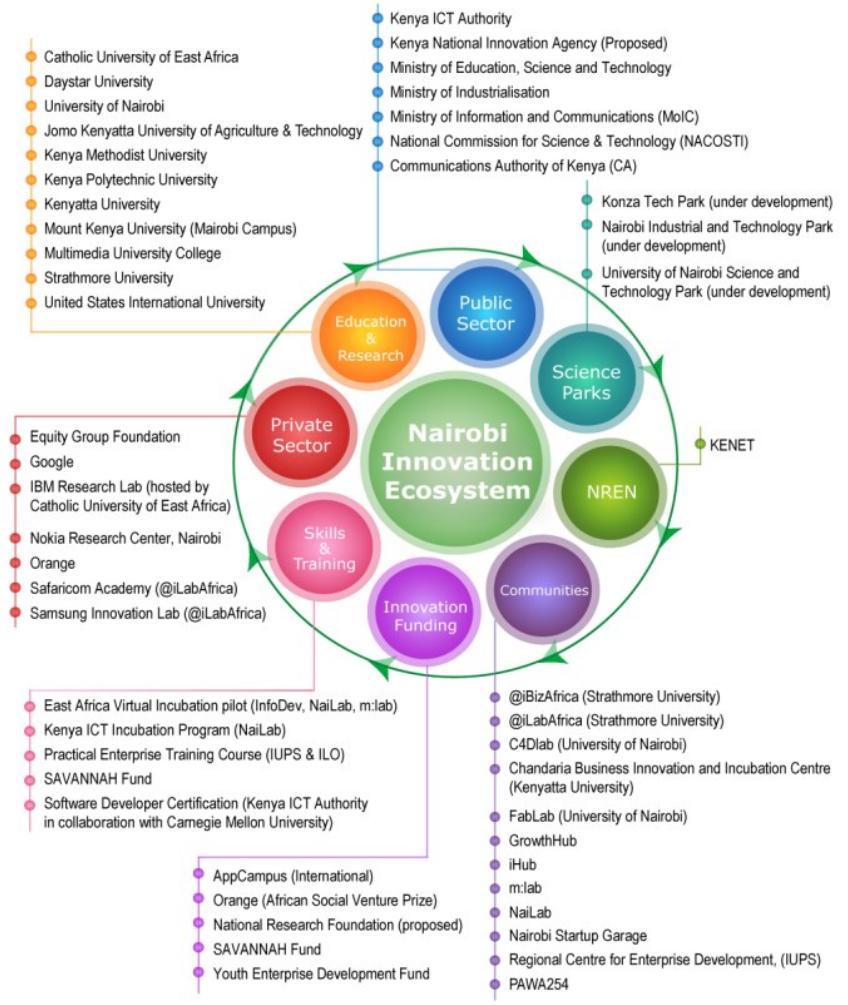

Figure 1. Nairobi Innovation Ecosystem [4]

These stakeholders have been previously discussed in [4].
While initially there was a quite rapid increase in Innovation and Entrepreneurship stakeholders in Nairobi from 2010, the main players have been quite consistent over the past four years. There is quite good collaboration between the public sector and other Innovation stakeholders. However, collaboration among other Innovation stakeholders and overall coordination of innovation activities is still at an early stage.

From the public sector, the Ministry of Education Science and Technology and National Commission for Science, Technology and Innovation (NACOSTI) have strong relationships with Kenya Education Network (KENET), all public and private Higher Education Institutions (HEIs) and research institutions and are important sources of research funding. The Kenya ICT Authority as implementing agency for the Ministry of ICT has an important role in funding Innovation and Entrepreneurship in the context of the Kenya Transparency and Communications Infrastructure project (KTCIP) and promoting private sector innovation. KENET as the National Education and Research Network (NREN) has a very important coordination role supporting over 150 campuses around Kenya as well as good collaboration with industry and Innovation Hubs. The proposed Kenya National Innovation Agency (KNIA) may play a key coordination role, with offices in all 47 Counties, and a mandate to institutionalise stakeholder linkages and promote incubation.

While it is important to align collaboration activities with national and organisational priorities, generally there is good collaboration between the public sector and other Innovation stakeholders, with the constraint of available human resources. Private sector stakeholders (Safaricom, Nokia, IBM, Ericsson, Samsung, Google, Nation Media Group) are quite active in Nairobi as Innovation Players (research labs) and supporting collaborative Innovation through sponsorship of events, training and equipment. This is particularly important for privately and community funded Innovation Spaces.

A significant number of HEIs (University of Nairobi, Kenyatta University, Strathmore University, Catholic University of East Africa, International University of Professional Studies, Jomo Kenyata University of Agriculture and Technology) actively support entrepreneurship through training programmes and Innovation Spaces / Research Labs to support Innovation actors develop their ideas. Due to their organisational mandates, scale and existing revenue streams, HEIs have the capacity to commit resources for collaboration, are well positioned to play a critical role in supporting Collaborative Open Innovation in Nairobi and assist in realising the objectives of Vision 2030.

@iLabAfrica (Strathmore University) has been pro-active in formally collaborating with industry (Safaricom, Vodafone, Ericsson, Samsung, Google, Deloitte Consulting), foundations (Clinton Health Access Initiative, IDEA Foundation) and universities (IT University of Copenhagen, MIT, Moi University, Egerton University, Mombasa Polytechnic, JKUAT and Mbabara University, Uganda). @iLabAfrica has co-organised events with iHub. iHub has cooperated with International University of Professional Studies in terms of entrepreneurship training, FabLab (University of Nairobi) in co-organised a Robotics Hackathon and co-organised events 
with Strathmore University such as the Open Data Workshop for journalists and application developers. iHub has a partnership with Stanford University and through m:lab East Africa collaborations with University of Nairobi, InfoDev, Microsoft and SEACOM. Strong corporate partnerships are in place with Nokia, Google, Samsung, Nation Media Group, Wananchi Group (Zuku), and Intel.

Based on interviews carried out in Nairobi, it is clear there is limited collaboration between Innovation Spaces and other Innovation Stakeholders. The exception is NaiLab, who secured a \$1.6 million Kenya ICT Incubation Program contract with Kenya ICT Authority (January 2013). There is limited differentiation between Innovation Spaces in Nairobi, in terms of activities, facilities, services offered and target community. This raises challenges associated with trust, potential conflicts of interest, identifying a win-win rationale for collaboration and achieving sustainability. There are also practical issues associated with prioritising the limited resources (personnel, time, funds) that exist.

The primary challenge for independent Innovation Spaces is to secure paying members or create a value proposition that justifies continued third party support. Most have limited membership or service income, and are dependent on grants to cover operating costs. This business model is increasingly unsustainable, with recent grant decisions providing funding to devise a sustainability model rather than support operations. Unless independent Innovation Spaces can reduce overheads through some form of consolidation (e.g. co-location, shared services) or tap into new types of members with capacity and willingness to pay, many are far from achieving sustainability. As a result, the ability of most independent Innovation Spaces to contribute resources towards collaboration is limited to inkind support (e.g. organising joint events).

Most current members of Innovation Spaces in Nairobi are still students or recent graduates. HEIs are also targeting students and recent graduates. Collaboration is harder to justify when it is not obvious where the complementarity or synergies between stakeholders exist. Except for entrepreneurs who enjoy parental support, work part-time or have some project income, the market is not yet mature enough to expect significant membership income. Some more forward thinking operators have developed additional revenue streams, which if successful can help bridge the gap between operating expenses and membership revenues.

It was envisaged when the Parliament approved the Science Technology and Innovation Bill in January 2013 that 2\% of GDP would be committed to fund national research and innovation grants through the Kenya National Research Council (KNRC). To date (June 2015) this has still not been realised and informally the Treasury is calling for a reduction in GDP contribution due to budgetary pressures. The potential impact of KNRC will depend on whether Innovation Spaces and other innovation stakeholders can also apply for grants, and the nature and timing of funding calls.

It would be beneficial if some formal coordination mechanism could be put in place at national level as a means to ensuring that all the positive energy that is being expended can collectively contribute to the realisation of the Vision
2030 goals. In many developed countries the concept of Centres of Excellence are based on the premise that there must be active collaboration between different Innovation Stakeholders and development of complementary activities. This should be seriously considered.

\section{DAR Es SALAAM InNOVATION ECOSYSTEM}

As outlined in Figure 2 below, since 2011 the Dar es Salaam Innovation Ecosystem has gradually expanded to include Innovation Spaces and Incubators.

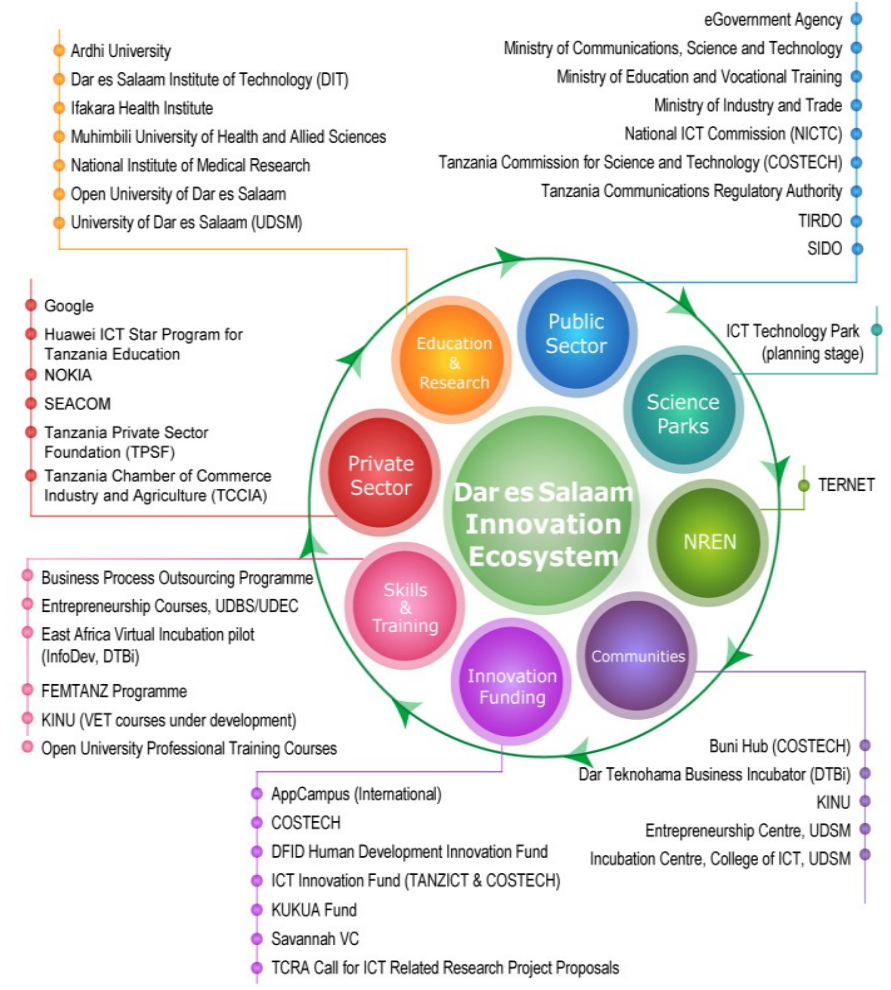

Figure 2. Dar es Salaam Innovation Ecosystem [4]

While the Innovation Ecosystem in Dar es Salaam is at an early stage of development, it is making good progress. Traditionally the main stakeholders have been the public sector and HEIs. During 2011 a number of new players commenced activities through collaboration with Info Dev in the case of Dar Teknohama Business Incubator (DTBi) and with the Ministry of Foreign Affairs of Finland in the case of Buni Hub (TANZICT Programme). KINU was formally launched by a group of entrepreneurs as an independent Innovation Space focused on pre-Incubation in 2012.

Tanzania Commission for Science and Technology $(\mathrm{COSTECH})$ has a key role in the Innovation Ecosystem due to its national coordination mandate. As a funding agency, COSTECH cooperates with all HEIs and other Research Institutes, provides internet access through TERNET NREN and hosts DTBi, TANZICT \& Buni Hub. COSTECH has included an Action Research requirement (engagement with industry or communities) within Masters and $\mathrm{PhD}$ programmes it supports financially. 
The Incubation Centre at the College of ICT, University of Dar es Salaam cooperates with the TANZICT Programme and many USDM students use the facilities in the Buni Hub. There is also regular cooperation between DTBi, Buni Hub (TANZICT Pre-Incubation Space) and KINU, mostly related to activities rotating between TANZICT and KINU and DTBi staff focused on identifying potential incubatees.

As in Nairobi, most members of Innovation Spaces in Dar es Salaam are students or recent graduates. For stakeholder collaboration to be sustainable, it is essential to identify complementarity and opportunities to maximise impact. KINU cooperates with TANZICT in providing training to women entrepreneurs and is working with Vocational Education and Training Authority (VETA) to certify training programmes.

At present the ecosystem is still fragmented with stakeholder cooperation driven by like-minded individuals and based on in-kind contributions (time, human resources). The financial capacity to invest in collaboration outside the public and HEI sector and donor funded projects is relatively low in Tanzania. There is limited national private sector involvement in Technology Innovation activities in Dar es Salaam apart from providing prizes for Hackathons or donating connectivity. It is very challenging for entrepreneurs to make a contribution towards operating costs, which means that Innovation Spaces must be creative in diversifying income streams. An Incubator opened by Mara Launchpad in Dar es Salaam in Q1 2013 closed within a year.

It would be beneficial to create a mechanism at national level to fund more formal cooperation since there is good potential for the different players to leverage synergies to reinforce their overall impact on supporting the Innovation community.

\section{KAMPALA INNOVATION ECOSYSTEM}

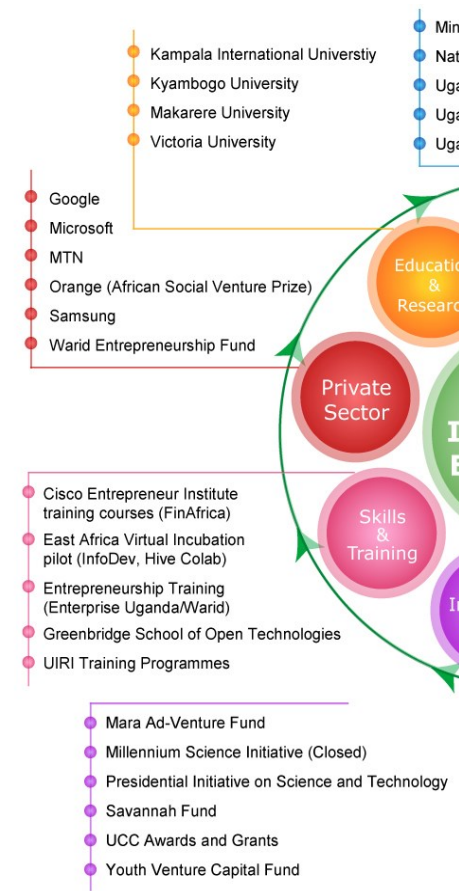

Figure 3. Kampala Innovation Ecosystem [4]

- Regional Science P (Proposed)
As outlined in Figure 3, like Nairobi and Dar es Salaam, the Innovation Ecosystem supporting Innovation and Technology Entrepreneurship in Kampala started to expand quite quickly beyond the traditional public sector, HEIs and NREN to include Innovation Spaces from 2009/2010.

Like Nairobi, there was rapid growth in Innovation and Entrepreneurship stakeholders in Kampala from 2009/2010 2012. However, this growth has slowed down considerably.

The difference in Kampala compared to Nairobi and Dar es Salaam is that the Innovation Spaces (Innovation Centre CIT; iLab@MAK; Mara Launchpad; The Hub Kampala; Outbox; HiveCoLab; Grameen Foundation AppLab Uganda; FinAfrica and Victoria University) made a concerted effort to collaborate and established the Business Innovation Consortium Kampala in 2012 (initiated by Grameen Foundation Apps Lab) as a mechanism to cooperate, share knowledge and attract entrepreneurs and investors. A joint awareness raising Start-Up event was organised in July 2012. While Governance subcommittees were established, it proved challenging to establish a common terms of reference for engagement with entrepreneurs. Cooperation has continued informally (e.g. coorganising a Hackathon, referring members for training). There is good collaboration between the public sector and other Innovation stakeholders. The Uganda National Council for Science and Technology (UNCST) is responsible for research coordination and as a funding agency has strong relationships with national research institutions. Uganda Communications Commission (UCC) supports the ICT education, research and private sectors through research grants, events and awards. The private sector is quite active with MTN, Orange, WARID having good Innovation programmes and organising competitions and mentoring to encourage Tech Entrepreneurs. While Innovation Space members in Nairobi and Dar es Salaam are mainly students and graduates, the community in Kampala includes more start-ups and existing businesses. Mara LaunchPad, The Hub Kampala and FinAfrica focus on existing businesses and start-ups, HiveCoLab, Outbox and Innovation Centre CIT support Pre-Incubation, students and graduates and Grameen Foundation AppsLab creates community solutions for commercialisation and deployment with MTN Uganda. and Technology
Incubation Centres (SPTIC)

Angels Hub (www.angelsinitiatives.org) took over the Mara LaunchPad incubation space in September 2013. Innovation Centre CIT, iLab@MAK (Makerere University) and Grameen AppLab have established strong public and private sector relationships over the years. HiveCoLab and Outbox work with members to identify commercial contracts they can fulfill and pay a percentage of the contract value to the Innovation Space for project management, which helps cover operating costs. HiveCoLab cooperates with other Innovation Spaces through AfriLabs and is an implementation partner for InfoDev East Africa Virtual Incubation pilot.

While there are limited financial resources available for collaboration in Kampala, Innovation Stakeholders cooperate well in leveraging in-kind contributions. For collaboration between all key Innovation Stakeholders (and particularly HEIs and Innovation Spaces) to be sustainable, it is essential to build linkages that result in a sustainable, "win-win" relationship. 


\section{ABSORPTION CAPACITY OF INNOVATION STAKEHOLDERS}

Based on the study undertaken, most key Innovation Stakeholders in Nairobi, Dar es Salaam and Kampala have a relatively high level of absorption capacity for innovative ideas. In terms of Heek's Ladder of ICT-Related Roles [12] they range from Intermediated Consumer to Innovator. The most successful innovations to date have been associated with cost-effective applications leveraging the high level of mobile penetration across Africa. Important sectors for ICT applications include Mobile Money, Agriculture, Health and Education. These range from incremental/progressive applications to transformational (e.g., Mobile Money).

While mobile subscribers in Kenya and Uganda have access to a variety of content-related services, in Tanzania smart phone penetration is low by comparison, with a far greater dependency on feature phones. In the health and education areas, this has significant content limitations, which in turn has impacted on the number of such services offered by operators. The significant reach of fibre optic networks, increasing broadband capabilities combined with Digital Broadcasting migration across all three countries opens new opportunities for local application and content development. Inclusive Innovation is required to provide low cost solutions for rural and deep rural areas and local content for Digital Broadcasting.

Having access to high quality Open Data Sets to support decision-making can provide a catalyst for developers to create innovative public and private sector tools. While progress has been achieved in Kenya and Tanzania, Uganda lags behind. However, as new services and broadband become more accessible, there is a need for capacity building to ensure people in urban, rural and deep rural environments all benefit. There is generally good public sector awareness in all three countries of the need to support Innovation and assist entrepreneurs to transition from concept to prototype and pilot to commercialisation. However, there is a need for tailored skills programmes supporting ICT-enabled Entrepreneurship and Innovation in HEIs and vocational training institutions.

Innovation absorption capacity can also be impacted by external factors including public sector procurement rules and regulatory environment. Traditional procurement rules are a significant challenge for Innovation take-up, particularly in the public sector. While entrepreneurs and early stage enterprises may have an innovative prototype or service which matches the technical requirements, they often cannot provide references or pass the mandatory financial viability checks.

Other key challenges identified during this study amongst many technology entrepreneurs include: insufficient access to entrepreneurship skills training (including team management); lack of sufficient awareness of the importance of NonDisclosure Agreements (NDAs) and Intellectual Property Rights (IPR); and access to quality mentoring and seed funding on equitable terms. Current skills gaps identified across all three cities based on face-to-face interviews include: lack of awareness of the importance of carrying out needs assessment; capacity to develop sustainable business models; User Interface design (particularly for mobile); business analyst skills; qualitative and quantitative research methodologies; and appreciation of Monitoring and Evaluation. There was a general consensus among stakeholders interviewed across all three cities that many applications and prototypes were being designed from a technical rather than an end-user consumer perspective. There was an acknowledgement that entrepreneurs need to be actively encouraged to identity potential clients, and co-design applications based on end-user requirements. Collaborative Open Innovation and Living Lab methodologies can assist in providing a mechanism for stakeholders to codesign innovations that address end-user requirements.

Given the rapidly changing environment, there is a need for a framework (such as ICT and Thematic Clusters) that brings HEIs, industry and policy makers together to share insights and co-create new applications and services. Currently no funds are allocated to such activities. ICT is still one of the least funded sectors in Uganda. Given that Innovation falls under several Ministries, there needs to be more harmonisation of priorities.

\section{VIII.DISCUSSION}

\section{A. Summary of Study Findings}

It is clear that the Governments of Kenya, Tanzania and Uganda recognise the critical role ICT-related Entrepreneurship can play in helping develop a Knowledge Economy and supporting sustainable socio-economic development. The basic enablers are in place in terms of proInnovation Policy and Regulatory frameworks, digital infrastructure has improved dramatically over the past decade and there is a significantly larger group of active Innovation Stakeholders. The ICT Policies in Tanzania and Uganda are currently under review. [2] measured the performance of 148 countries in leveraging ICT to boost competitiveness and wellbeing based on four main sub-indices: 1. Enabling Environment (Political and regulatory environment; business and innovation environment); 2. Readiness of key stakeholders to use ICT infrastructure and digital content (Infrastructure and digital content, Skills, Affordability); 3. ICT Usage (individuals, businesses, government) and 4. Economic and social impact of ICTs. [2] ranks Kenya in 92nd place, Uganda in 115th place and Tanzania in 125th place. There is a common focus on leveraging mobile technology, taking advantage of high levels of mobile penetration and telecoms competition in East Africa. This finding agrees with Heeks' premise on the importance of supporting mobile users [13, p23]. It is clear there is still considerable scope to increase local content provision, particularly in Tanzania where content offering as part of mobile subscriptions are currently limited. Sections 4 6 provided an overview of Innovation Stakeholders and current level of collaboration. It is extremely positive that the Innovation Ecosystems in Nairobi, Dar es Salaam and Kampala now include the private sector and Innovation Spaces, as well as the traditional actors (e.g. public, education and research sectors). It is interesting that this trend started in Uganda in 2009/2010, followed quickly by Kenya and then Tanzania.

The learning-centered approach [14] is important in the context of collaboration and enhancing Innovation capacity, as presented in this paper. As outlined by [14] it is necessary to have both experimentation and collaboration to improve the learning experience, benefit from collective expertise and knowledge that other Innovation Stakeholders can contribute, 
to strengthen the overall Innovation Ecosystem. Applying the Ladder of ICT-related Roles [13] can facilitate stakeholder and community segmentation to better understand the capacity of Innovation Ecosystem Stakeholders as well as providing a roadmap towards strengthening the overall Innovation Ecosystem. Innovation Stakeholders in Nairobi, Dar es Salaam and Kampala range from Intermediate Consumer to Innovation. Innovation Spaces are primarily supporting the following roles: Active Users; Producers; Workers; Entrepreneurs; and Innovators. Section 7 highlights that while there is a relatively high level of absorption capacity in the three cities studied, there are also common challenges that impact directly on innovation generation and take-up. These include: public procurement rules; skills development; research methods capacity (including needs requirements gathering); and business model development. While it is positive that there are now dynamic Innovation Ecosystems in these cities, there is an urgent need to actively encourage all key stakeholders to actively collaborate and share experiences to strengthen the overall Innovation Ecosystem. There are practical and cultural challenges associated with coordination and collaboration, as ultimately these require trust relationships.

It is necessary for the public sector as the primary funding source for the National Innovation System to develop support mechanisms that facilitate establishment of more formal and sustainable coordination platforms to build momentum and ensure that good practices, achievements and impact are recognised and exploited. In the case of Kenya for example, this could be explored in the context of establishing KNIA.

\section{B. Alignment of Study Results with GII 2014}

The study findings are consistent with the premise that to identify relevant strategies and polices that energise innovators [9, p6] "it is important to learn more about what happens at the intersection of people, technology, financing, policy and institutions" and "Improving skills is one of the most important ways to raise innovation, productivity and economic growth."

The overall GII 2014 findings confirm that the Innovation Ecosystems in Kenya and Uganda are more mature than Tanzania, which is consistent with the findings of this study. It is clear that investments in Kenya and Uganda in infrastructure and capacity building are bearing fruit. This is reflected in the classification of Kenya and Uganda by GII 2014 as Innovation Learners - defined as (a) "economies that perform at least $10 \%$ higher than expected for their level of GDP" (p11). "SubSaharan Africa now comprises nearly 50\% of the innovation learner economies" and "most significant improvement of all regions in the GII rankings". Kenya is classified by GII 2014 as an Efficient Innovator, while the Innovation Efficiency Ratios for Uganda and Tanzania fall below the median $(0.74)$ of economies analysed. However, based on the findings of the GII 2014 study, both Nairobi and Kampala are making considerable progress in strengthening the underlying factors related to Human Capital and Research, Infrastructure and Business Sophistication. Tanzania has made considerable investment in infrastructure and strengthening institutional capacity, particularly over the last five years. Based on the current level of innovation and ICT-related entrepreneurship activity in Dar es Salaam, we expect continued improvement in
Tanzania's Innovation Output Sub-Index Score which currently lags behind Uganda and Kenya, and explains the significantly lower Innovation Efficiency Ratio.

While the authors agree with [-9, p46], that the "level and standard of education and research activity in a country are prime determinants of the innovation capacity of a nation", we perceive closer Human Capital and Research capacity between Nairobi and Kampala, than reflected in GII 2014 between Kenya and Uganda. Furthermore, [9, p49] states that "Innovation linkages and public/private/academic partnerships are essential to innovation", this study finds significantly stronger Innovation Linkages and Knowledge Absorption capacity in Nairobi, Dar es Salaam and Kampala than reflected in the Pillar 5 sub-scores for Kenya, Tanzania and Uganda. While the lower Knowledge Workers score for Tanzania compared to Uganda is consistent, the even lower score given to Kenya is inexplicable on the basis of the situation in Nairobi.

\section{Factors Potentially Influencing Differences}

Several factors can explain differences between findings of this study of ICT-related collaboration and innovation capacity of Nairobi, Dar es Salaam and Kampala [9].

First, the results of this study are based on qualitative data collection, leveraging face-to-face semi-structured interviews with senior representatives from a broad range of innovation stakeholders to gather high quality data. Because of its global geographic scope (143 economies), [9] relies primarily on quantitative data gathered from over 30 sources. GII recognizes the potential risks associated with not adequately capturing "some important qualitative aspects of innovation policies and processes ... within the GII model" (p37). It addresses this factor by including specific chapters in each report which "provide additional details on successful strategies for leveraging the human factor in innovation."

Second, the findings presented in this paper are a sub-set of the results of a more comprehensive study which is also addressed in part in [3]. The information used is as complete as possible, although clearly not as comprehensive in terms of data points as [9]. The difficulty for [9] is that complete information is not always available to inform the Innovation Input and Innovation Output Sub-Index scores (and rank) of each economy. As explained earlier, in the context of this paper's focus on assessing potential ICT-related Collaboration and Innovation Capacity, Pillar 2 and Pillar 5 (and contributing indicators) of the Input Sub-Index Pillars are particularly relevant. When calculating Pillar 2 (Human Capital and Research), while all sub-indicators affecting the Education indicator score are available for Kenya (p207), Tanzania ((p264) and Uganda (p271), Kenya and Tanzania Tertiary Education indicator comparable data is missing, impacting their score and rank. When calculating Pillar 5 (Business Sophistication), comparable data for Kenya and Tanzania is missing for the Knowledge Workers indicator score, while data for Tanzania and Uganda is missing for Innovation Linkages indicator. The effect of even small data gaps impact on accurately calculating the respective Innovation Efficiency Ratio (Output Sub-Index/Input Sub-Index). 
Third, to a degree this is comparing apples with pears - GII 2014 reflects the overall Kenyan, Ugandan and Tanzanian economies, while this study is focused on innovation ecosystem stakeholders in Nairobi, Dar es Salaam and Kampala. It could be expected that the level of Innovation Capacity in a major city would be higher than across a country.

\section{CONCLUSION}

This paper presents a sub-set of results from a comprehensive baseline analysis of Innovation Ecosystems in Nairobi, Dar es Salaam and Kampala with a focus on assessing the current level of ICT-related Collaboration and Innovation Absorption capacity. As outlined in [9, p6], "it is important to learn more about what happens at the intersection of people, technology, financing, policy and institutions", "Improving skills is one of the most important ways to raise innovation, productivity and economic growth" and "Innovation linkages and public/private/academic partnerships are essential to innovation" (p49). Relevant literature is outlined, including the GII 2014 framework [9], to provide an overall context against which to compare the study findings presented. Sens' Capability Approach [15], the learning-centred approach [14] and Heeks Ladder of ICT-Related Roles [13] are well aligned with the findings of this paper. An overview of the innovation stakeholders in Nairobi, Dar es Salaam and Kampala and their current collaboration capacity, interactions and challenges, is complemented by an assessment of the level of absorption capacity and common challenges. It is very positive that due to a significant investment in digital infrastructure and a proinnovation policy and regulatory framework, the Innovation Ecosystems in Nairobi, Dar es Salaam and Kampala have considerably expanded, incorporating new national and international Innovation Stakeholders over the last five years. This will increase the probability of successfully realising the objectives of National Development Plans (Kenya - Vision 2030, Uganda - Vision 2040, Tanzania - Vision 2025), as well as addressing current challenges related to youth and graduate unemployment and developing a skilled workforce. Now that credible Innovation Ecosystems are in place, the real challenge begins - how to achieve sustainability and build an Innovation and Entrepreneurial Culture through an Open Collaboration ethos that leverages the insight and contributions that different stakeholder groups (including end-user communities) can make. It is requirement for the public sector to take an active leadership role in enabling this process, and encouraging and facilitating multi-stakeholder collaboration through dedicated support mechanisms. It is also necessary to put specific plans in place to address challenges identified in Section 7.1 that impact both absorption capacity and innovation take-up. Collaboration is harder to justify when it is not obvious where potential synergies exist between stakeholders. It is necessary to identify potential "win-win" scenarios that will help foster the level of trust and openness required for a Collaborative Open Innovation culture to become operational.

Future research will focus on comparing these results with the situation in other IST-Africa countries (and internationally) and proposing a model (and actionable recommendations) to strengthen developing country innovation ecosystems.

\section{ACKNOWLEDGEMENTS}

This research was co-funded by the European Commission under IST-Africa (FP7 Contract 288691). The authors wish to acknowledge invaluable contributions from IST-Africa Partners, IST-Africa Workshop participants, and in particular, senior stakeholders in Nairobi, Kampala and Dar es Salaam who agreed to be interviewed and generously shared insight.

\section{REFERENCES}

[1] Alkire, S. (2002). Dimensions of Human Development, World Development, Volume 30, Issue 2, February 2002, pp. 181-205

[2] Bilbao-Osario, B., Dutta, S. and Lanvin, B (Editors) (2014), The Global Information Technology Report, World Economic Forum and INSEAD, ISBN: 978-92-95044-63-0 www3.weforum.org/docs/WEF_GlobalInformationTechnology_Report_2014.pdf

[3] Creswell, J.W. (2007) Qualitative inquiry \& research design: Choosing among five approaches (2nd ed.), Thousand Oaks, CA: Sage.

[4] Cunningham P., Cunningham M., Ekenberg L. 2014. Baseline Analysis of 3 Innovation Ecosystems in East Africa. International Conference on Advances in ICT for Emerging Regions (ICTer2014), IEEE Xplore, DOI: 10.1109/ICTER.2014.7083895

[5] Cunningham P., Cunningham M., et al 2014. Guide to Bilateral \& Multilateral Cooperation Agreements Supporting ICT/STI-related Activities in IST-Africa Partner Countries. ISBN: 978-1-905824-42-7 www.ist-africa.org/home/files/IST-Africa_BilateralCooperation_310114.pdf

[6] Cunningham P., Cunningham M., et al 2014. Guide to ICT Initiatives and Research Capacity in IST-Africa Partner Countries. ISBN: 978-1905824-41-0 - www.ist-africa.org/home/files/ISTAfrica_ICTInitiatives_ResearchCapacity_310114.pdf

[7] Denscombe, M. (2010), The Good Research Guide, Open University Press

[8] DTI (Department of Trade and Industry) (1998) White Paper: Our Competitive Future - building the knowledge-economy economy. London: HMSO

[9] Dutta, S., Lanvin, B., Wunsch-Vincent, S. (Eds.) The Global Innovation Index 2014: The Human Factor in Innovation. Cornell University (US), INSEAD (France) and WIPO (Switzerland). ISBN 978-2-9522210-6-1

[10] Furman, J. L.; Porter, M. E.; Stern, S., 2002. "The Determinants of National Innovation Capacity”, Research Policy 31, pp. 899-933.

[11] Heeks, R. \& Molla, A., (2009). Impact Assessment of ICT-forDevelopment Projects: A Compendium of Approaches, Development Informatics Group, University of Manchester, 2007. ISBN: 978-1905469-03-1

[12] Heeks, R. (2014, July 08). Understanding ICT4D and Capabilities via User Roles. ICT4D Blog, Retrieved [September 27, 2014] from http://ict4dblog.wordpress.com/2014/07/08/understanding-ict4d-andcapabilities-via-user-roles/

[13] Heeks, R (2014) ICTs and Poverty Eradication: Comparing Economic, Livelihoods and Capabilities Models, Centre for Development Informatics, University of Manchester, UK www.seed.manchester.ac.uk/subjects/idpm/research/publications/wp/di/di-wp58/

[14] Sabel C. and Reddy S. (2007) Learning to Learn: Undoing the Gordian Knot of Development Today, Challenge Journal, Sharpe, 2007, DOI 10.2753/0577-5132500505

[15] Sen, A. (1999). Development as Freedom, Oxford University Press

[16] Thapa, D., Sein, M. \& Saebo. O. (2012). Building collective capabilities through ICT in a mountain region of Nepal: where social capital leads to collective action. Information Technology for Development, Volume 18, Issue 1

[17] UNDP Human Development Report 1990 